\section{Neuroretinal alterations in the early stages of diabetic retinopathy in patients with type 2 diabetes mellitus}

P Carpineto ${ }^{1,5}$, L Toto ${ }^{1,5}$, R Aloia ${ }^{1}$, V Ciciarelli ${ }^{1}$, E Borrelli ${ }^{1}$, E Vitacolonna ${ }^{2}, \mathrm{M}$ Di Nicola ${ }^{3}$,

L Di Antonio ${ }^{1}$ and R Mastropasqua ${ }^{4}$

\begin{abstract}
Purpose To study neuroretinal alterations in patients affected by type 2 diabetes with no diabetic retinopathy (DR) or mild nonproliferative diabetic retinopathy (NPDR) and without any sign of diabetic macular edema.

Patients and methods In total, 150 type 2 diabetic patients with no (131 eyes) or mild NPDR (19 eyes) and 50 healthy controls were enrolled in our study. All underwent a complete ophthalmologic examination, including Spectral-Domain optical coherence tomography (SD-OCT). Ganglion cell-inner plexiform layer (GC-IPL) and retinal nerve fiber layer (RNFL) thickness values were calculated after automated segmentation of SD-OCT scans.
\end{abstract}

Results Mean best-corrected visual acuity was $0.0 \pm 0.0$ LogMAR in all the groups. Mean GC-IPL thickness was $80.6 \pm 8.1 \mu \mathrm{m}$ in diabetic patients and $85.3 \pm 9.9 \mu \mathrm{m}$ in healthy controls, respectively $(P=0.001)$. Moreover, evaluating the two different diabetic groups, GC-IPL thickness was $80.7 \pm 8.1 \mu \mathrm{m}$ and $79.7 \pm 8.8 \mu \mathrm{m}$ in no-DR and mild-NPDR group $(P=0.001$ and $P=0.022$ compared with healthy controls, respectively). Average RNFL thickness was $86.1 \pm 10.1 \mu \mathrm{m}$ in diabetes patients and $91.2 \pm 7.3 \mu \mathrm{m}$ in controls, respectively $(P=0.003)$. RNFL thickness was $86.4 \pm \mathbf{1 0 . 2} \mu \mathrm{m}$ in no-DR group and $84.1 \pm 9.4 \mu \mathrm{m}$ in mild-NPDR group $(P=0.007$ and $P=0.017$ compared with healthy controls, respectively).

Conclusion We demonstrated a significantly reduced GC-IPL and RNFL thickness values in both no-DR and mild-NPDR groups compared with healthy controls. These data confirmed neuroretinal alterations are early in diabetes, preceding microvascular damages.
Eye (2016) 30, 673-679; doi:10.1038/eye.2016.13; published online 12 February 2016

\section{Introduction}

Diabetic retinopathy (DR) is a major complication of diabetes and the leading cause of decreased vision in working-age people. ${ }^{1} \mathrm{DR}$ is primarily a vascular disease, in which structural changes in the retinal vessel endothelium leads to breakdown of the blood-retina barrier and increased vascular permeability. ${ }^{2}$

By contrast, retinal functional impairment may occur early in the course of diabetes and in patients without any signs of DR, suggesting a role for neuroretinal damage in the pathogenesis of DR. ${ }^{3,4}$ These data suggest that diabetes causes vision impairment associated with alterations in the electrophysiological and psychophysiological measurements of retinal function. ${ }^{3,4}$ These changes precede evident vascular lesions associated with DR and suggest that diabetes compromises the function of retinal neuronal cells before the blood-retinal barrier is significantly altered. Moreover, neuroretinal damage is also demonstrated by the structural changes in the retina of the diabetic patient. Barber et $a l^{5}$ in an autopsy study, showed a reduction of the ganglion cell-inner plexiform layer (GC-IPL) in diabetic patients without any signs of DR.

The introduction of optical coherence tomography (OCT) has allowed the imaging and measuring of retinal thickness with high accuracy, and several authors showed decreased retinal thickness in diabetic patients with no or mild DR compared with normal controls. ${ }^{6-9}$ The high resolution of spectral domain-OCT (SD-OCT) allows thickness measurement of all individual retinal layers after automated segmentation.
${ }^{1}$ Ophthalmology Clinic, Department of Medicine and Science of Ageing, High-Tech National Center in Ophthalmology, University G. D'Annunzio Chieti-Pescara, Chieti, Italy

${ }^{2}$ Diabetic Unit, Department of Medicine and Science of Ageing, University G.

D'Annunzio Chieti-Pescara, Chieti, Italy

${ }^{3}$ Center of Excellence on Aging and Department of Biomedical Science, University 'G. D'Annunzio' of Chieti, Laboratory of Biostatistics, Chieti, Italy

${ }^{4}$ Ophthalmology Unit, Department of Neurological, Neuropsychological, Morphological and Movement Sciences, University of Verona, Verona, Italy

Correspondence: L Toto, Ophthalmology Clinic, Department of Medicine and Aging Sciences, High-Tech National Center in Ophthalmology, University G. D'Annunzio ChietiPescara, Via dei Vestini, Chieti, 66100, Italy Tel: +39871 358410; Fax: +39871357294. E-mail: totolisa@hotmail.com

${ }^{5}$ These authors are joint first authors.

Received: 24 July 2015 Accepted in revised form: 20 October 2015 Published online: 12 February 2016 
In this study, we assessed the retinal GC-IPL and retinal nerve fiber layer (RNFL) thickness values using SD-OCT in type 2 diabetic patients with no or mild nonproliferative diabetic retinopathy (NPDR) and without any signs of diabetic macular edema. The objective of this study was to determine whether type 2 diabetes causes the thinning of these retinal layers in patients with no or mild NPDR or any signs of diabetic macular edema.

\section{Materials and methods}

We enrolled 150 type 2 diabetic patients who consecutively presented to the Ophthalmology Clinic of the University of Chieti-Pescara between January 2013 and July 2014. The study adhered to the tenets of the Declaration of Helsinki and was approved by the local Ethics Committee. Informed consent was obtained from all patients before enrollment.

Baseline evaluation included a detailed medical history regarding the general health status, systemic hypertension and blood levels of glycated hemoglobin (HbA1c). All patients underwent a complete ophthalmologic examination, including assessment of best-corrected visual acuity (BCVA) using the Early Treatment Diabetic Retinopathy Study chart, slit-lamp biomicroscopy, intraocular pressure measurement with Goldmann applanation tonometry, central corneal pachimetry, visual field test (Humphrey visual field test 30-2, Carl Zeiss Meditec Inc., Dublin, CA, USA), and indirect fundus ophthalmoscopy with a 78-diopter lens and SD-OCT.

Criteria for inclusion were as follows: (1) age $\geq 18$ years old; (2) diagnosis of type 2 diabetes; (3) no sign of retinopathy or presence of NPDR corresponding to grade 20 on the Early Treatment Diabetic Retinopathy Study (ETDRS) scale; ${ }^{10}$ and (4) BCVA of at least 0.1 LogMAR.

Exclusion criteria were as follows: (1) evidence of diabetic macular edema on fundus biomicroscopy or on SD-OCT images (central subfield thickness > 250 microns); (2) previous ocular surgery, including refractive surgery, or retinal laser treatment; (3) any retinopathy secondary to causes other than diabetes, including the presence of a maculopathy, epiretinal membrane, or vitreomacular traction syndrome; (4) any optic neuropathy, including glaucoma, or any condition increasing the risk of secondary glaucoma (eg, pigment dispersion syndrome or pseudoexfoliation syndrome); (5) any neurodegenerative diseases known to influence RNFL thickness ${ }^{11,12}$ (eg, Alzheimer's or Parkinson's diseases); (6) refractive error $>3$ diopters; (7) intraocular pressure $>21 \mathrm{~mm} \mathrm{Hg}$; (8) visual field alteration (subjects were declared healthy if the visual field mean deviation, pattern SD and the glaucoma hemifield test were all within normal limits; visual fields were considered reliable if fixation loss and false-negative and false-positive results were $<30 \%$ ); and (9) significant media opacities.

A control group of 50 subjects, homogenous for age and sex, was also included in the current analysis. All control subjects also underwent a complete ophthalmologic examination, including a visual field test and SD-OCT.

\section{Imaging}

Patients were tested using a Cirrus SD-OCT (Carl Zeiss Meditec Inc.), a commercially available device with a scan speed of 27000 axial scans per second and an axial resolution of $5 \mu \mathrm{m}$. All scans were acquired by the same operator after pupil dilation using eye drops containing $0.5 \%$ tropicamide and $0.5 \%$ phenylephrine hydrochloride. Cirrus SD-OCT was used to acquire two macular scans using the macular cube $512 \times 128$ scan protocol and the Optic Disc Cube $200 \times 200$ protocol. The GCA algorithm, incorporated into the Cirrus SD-OCT software version 6.5, was used to process and measure the thickness of the macular GC-IPL within a $14.13-\mathrm{mm}^{2}$ elliptical annulus area centered on the fovea. The GCA algorithm automatically segmented the GC-IPL based on the three-dimensional data generated from the macular cube $512 \times 128$ scan protocol (Figure 1). The average, minimum and six sectoral GC-IPL thickness values (supero-temporal (ST), superior (S), supero-nasal (SN), infero-nasal (IN), inferior (I), and infero-temporal (IT)) were measured from the elliptical annulus centered on the fovea. To evaluate RNFL thickness, Cirrus SD-OCT
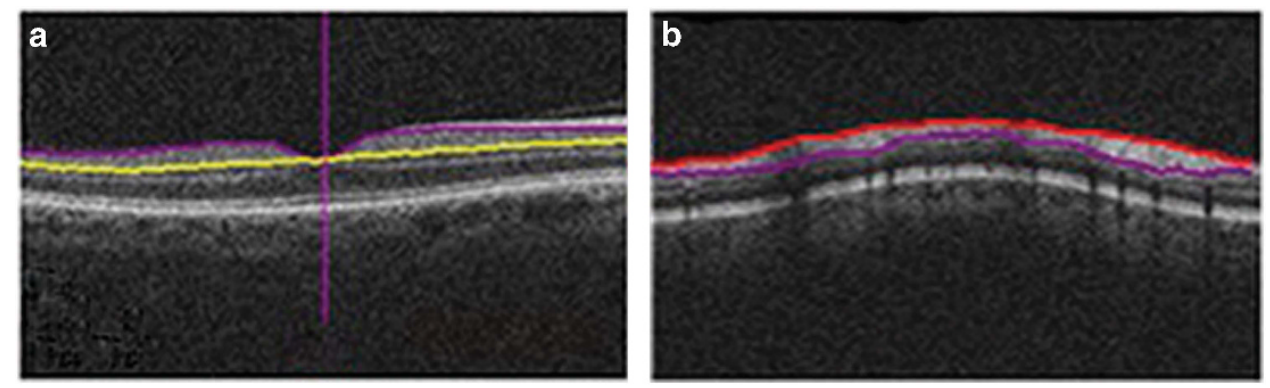

Figure 1 Automated segmentation, by regular Cirrus algorithm, identifies the ganglion cell-inner plexiform layer (a) and the retinal nerve layer thickness (b). 
algorithms identify the optic disc and automatically place a calculation circle with a 3.46-mm diameter evenly around it. Layer-seeking algorithms determine the RNFL inner (anterior) boundary and RNFL outer (posterior) boundary for the entire cube, except the optic disc (Figure 1). The system extracts data from the cube 256A-scan samples along the path of the calculation circle. The process results in a T, S, N, I, and T profile map. A detailed description of the algorithm has been presented in detail. ${ }^{13}$

During the scanning, the subject's pupil was first centered and focused in the iris viewport and the linescanning ophthalmoscope with 'auto focus' mode was then used to optimize the view of the retina. The 'center' and 'enhance' modes were used to optimize the Z-offset and scan polarization, respectively, for the OCT scan to maximize the OCT signal. After each capture, the motion artifact was checked with the line-scanning ophthalmoscope image with an En Face OCT overlay. Rescanning was performed if a motion artifact, indicated by blood vessel discontinuity, was detected.

Only good-quality scans, defined as scans with signal strength $\geq$ six, were used for the analysis.

\section{Statistical analysis}

RNFL and GC-IPL measurements were compared using the one-way analysis of variance, followed by the Bonferroni post hoc test. Student's $t$-test was used to compare quantitative variables, such as diabetes duration and $\mathrm{HbA1c}$ value. Skewness and Kurtosis values were calculated to assess the normal distribution of the variables. Furthermore, categorical variables were compared with the $\chi^{2}$-test. Finally, Pearson's correlation was performed to evaluate the linear correlation between variables (RNFL and GC-IPL).

All statistical analyses were performed using MedCalc version 8.1 for Windows (MedCalc, Mariakerke, Belgium), and a $P$-value $<0.05$ was considered statistically significant.

\section{Results}

A total of 150 eyes from 150 type 2 diabetic patients (94 males, 56 females; mean age $60.9 \pm 8.3$ years, range 31-83 years) were tested. Of these, 131 patients did not have any signs of DR (no-DR group), and the remaining 19 patients were affected by mild NPDR (mild-NPDR group). A control group consisting of 50 subjects, homogenous for age and sex (30 males, 20 females; mean age $60.0 \pm 8.4$ years, range $25-80$ years), was selected for statistical comparisons (Table 1). The BCVA was $0.0 \pm 0.0$ LogMAR in all groups. The mean refractive error was $0.2 \pm 1.5$ diopters in diabetic patients and $0.1 \pm 1.3$ diopters in healthy controls $(P=0.466)$.

Patients affected by diabetes reported a mean duration of disease of $7.1 \pm 6.6$ years $(6.4 \pm 5.6$ years and $12.8 \pm 10.4$ years for no-DR and mild-NPDR patients, respectively). The mean $\mathrm{HbA} 1 \mathrm{c}$ level was $7.4 \% \pm 1.3 \%, 7.4 \% \pm 1.2 \%$, and $8.0 \% \pm 1.3 \%$ in diabetic patients, no-DR group, and mildNPDR group, respectively (Table 1).

Additional demographic and clinical characteristics of the enrolled subjects are reported in Table 1.

The GC-IPL and RNFL thickness measurements were tested for all diabetic and control eyes. Upon SD-OCT

Table 1 Characteristics of diabetic patients and controls

\begin{tabular}{|c|c|c|c|c|c|}
\hline & \multicolumn{3}{|c|}{ Diabetic patients $(\mathrm{n}=150)$} & \multirow[t]{2}{*}{ Controls $(\mathrm{n}=50)$} & \multirow[t]{2}{*}{ P-value } \\
\hline & Overall patients & no-DR group $(\mathrm{n}=131)$ & mild-NPDR group $(\mathrm{n}=19)$ & & \\
\hline Age (years) & $60.9 \pm 8.3$ & $60.6 \pm 8.3$ & $62.7 \pm 8.4$ & $60.0 \pm 8.4$ & $0.158^{\mathrm{a}}$ \\
\hline Gender, n (\%) & & & & & $0.540^{\mathrm{b}}$ \\
\hline Male & $94(62.7)$ & $80(61.1)$ & $14(73.7)$ & $30(60.0)$ & \\
\hline Female & $56(37.3)$ & $51(38.9)$ & $5(26.3)$ & $20(40.0)$ & \\
\hline Duration of diabetes (years) & $7.1 \pm 6.6$ & $6.4 \pm 5.6$ & $12.8 \pm 10.4$ & & $<0.001^{\mathrm{c}}$ \\
\hline BCVA & $0.0 \pm 0.0$ & $0.0 \pm 0.0$ & $0.0 \pm 0.0$ & $0.0 \pm 0.0$ & \\
\hline $\mathrm{HbA} 1 \mathrm{c}(\%)$ & $7.4 \pm 1.3$ & $7.4 \pm 1.2$ & $8.0 \pm 1.3$ & & $0.050^{c}$ \\
\hline IOP $(\mathrm{mmHg})$ & $14.4 \pm 2.5$ & $14.5 \pm 2.5$ & $14.1 \pm 2.8$ & $14.3 \pm 2.2$ & $0.758^{\mathrm{a}}$ \\
\hline Refractive error & $0.24 \pm 1.47$ & $0.28 \pm 1.53$ & $-0.02 \pm 1.03$ & $0.05 \pm 1.23$ & $0.466^{\mathrm{a}}$ \\
\hline Treatment, n (\%) & & & & & $<0.001^{\mathrm{b}}$ \\
\hline Diet & $14(9.3)$ & $12(9.2)$ & $2(10.5)$ & & \\
\hline OHA & $116(77.3)$ & $106(80.9)$ & $10(52.6)$ & & \\
\hline Insulin & $13(8.7)$ & $11(8.4)$ & $2(10.6)$ & & \\
\hline INS+OHA & $7(4.7)$ & $2(1.5)$ & $5(26.3)$ & & \\
\hline
\end{tabular}

Abbreviations: BCVA, best-corrected visual acuity (logMAR (logarithm of the minimum angle of resolution)); HbA1c, glycated hemoglobin; INS, Insulin; IOP, intraocular pressure; mild-NPDR group, diabetes patients with signs of mild nonproliferative diabetic retinopathy (NPDR); $n$, number of patients; no-DR group, diabetes patients without any sign of diabetic retinopathy (DR); OHA, oral hypoglycaemic agent. ${ }^{\mathrm{a}}$ One-way ANOVA test. ${ }^{\mathrm{b}} \chi^{2}$-test. 'Student's $t$-test for unpaired data no-DR group $v$ s mild-NPDR group. Statistical significant $P$-values are written in bold. 
examination, the average RNFL thickness was $86.1 \pm 10.1 \mu \mathrm{m}$ and $91.2 \pm 7.3 \mu \mathrm{m}$ in diabetic patients and controls, respectively $(P=0.003)$. Furthermore, for the two different groups of diabetic patients, the average RNFL thickness was $86.4 \pm 10.2$ in the no-DR group and $84.1 \pm 9.4$ in the mild-NPDR group $(P=0.977)$. Moreover, both the no-DR group and mild-NPDR group showed a statistically significant difference in the average RNFL thickness compared with the control group $(P=0.007$ and $P=0.017$, respectively) (Table 2). Moreover, the RNFL thickness was significantly different between the diabetic patients and controls in the superior and temporal quadrants (Table 2).

The average GC-IPL thickness was $80.6 \pm 8.1 \mu \mathrm{m}$ and $85.3 \pm 9.9 \mu \mathrm{m}$ in diabetic patients and controls, respectively $(P=0.001)$. Moreover, for the two different diabetic groups, the GC-IPL thickness was $80.7 \pm 8.1 \mu \mathrm{m}$ and $79.7 \pm 8.8 \mu \mathrm{m}$ in the no-DR and mild-NPDR group, respectively $(P=1.000)$. Furthermore, both the no-DR group and mild-NPDR group showed a statistically significant difference in the mean GC-IPL thickness compared with the control group ( $P=0.001$ and $P=0.022$, respectively) (Table 2$)$. In addition, in all quadrants, the mean GC-IPL thickness was significantly difference between the diabetic patients and controls (Table 2).

Additional GC-IPL and RNFL thickness analyses are shown in Table 2.

To improve the data analysis, we selected patients in whom the SD-OCT software did not show abnormalities in both the RNFL and GC-IPL analysis. In these patients, the analysis did not output any average or sectorial thickness reduction for both RNFL and GC-IPL. Only 88 of 150 patients had no abnormalities on the RNFL and GC-IPL analysis. All selected patients belonged to the no-DR group. This selected group showed an average thickness of $86.5 \pm 9.7 \mu \mathrm{m}$ and $80.3 \pm 8.1 \mu \mathrm{m}$ in RNFL and GC-IPL layers, respectively. Both the RNFL thickness and GC-IPL thickness were reduced in this group compared with the control group ( $P=0.015$ and $P=0.001$, respectively) (Table 3). All patients affected by mild NPDR had alterations either in the RNFL or GC-IPL analysis.

The Pearson test showed that the RNFL thickness was directly correlated with the GC-IPL thickness in diabetic patients $\left(R^{2}=0.305, P<0.001\right)$. We also found a direct correlation between the average RNFL thickness and $\mathrm{HbA1c}$ value $\left(R^{2}=0.162, P=0.048\right)$. This correlation reached statistical significance in the inferior RNFL sector. Moreover, we found no correlation between RNFL and GC-IPL thickness values and diabetes duration (Table 4).

\section{Discussion}

In this cross-sectional study using SD-OCT, we investigated the GC-IPL and RNFL thickness values in asymptomatic type 2 diabetic patients with no or mild NPDR, corresponding to grade 20 or 35 on the ETDRS scale, without diabetic macular edema. Overall, the current analysis revealed a significant reduction of the mean GC-IPL thickness and RNFL thickness in type 2 diabetic patients with no or mild NPDR compared with

Table 2 Ganglion cell-inner plexiform layer and retinal nerve fiber layer thicknesses in diabetic patients and controls

\begin{tabular}{|c|c|c|c|c|c|c|c|c|}
\hline & \multicolumn{3}{|c|}{ Diabetic patients $(\mathrm{n}=150)$} & \multirow{2}{*}{$\begin{array}{l}\text { Controls } \\
(\mathrm{n}=50)\end{array}$} & \multicolumn{4}{|c|}{ P-value } \\
\hline & $\begin{array}{l}\text { Overall } \\
\text { patients }\end{array}$ & $\begin{array}{c}\text { no-DR } \\
\text { group } \\
(\mathrm{n}=131)\end{array}$ & $\begin{array}{l}\text { mild-NPDR } \\
\quad \text { group } \\
(\mathrm{n}=19)\end{array}$ & & $\begin{array}{l}\text { Diabetic } \\
\text { patients vs } \\
\text { controls }\end{array}$ & $\begin{array}{l}\text { No-DR } \\
\text { group vs } \\
\text { controls }\end{array}$ & $\begin{array}{l}\text { mild-NPDR } \\
\text { group vs } \\
\text { controls }\end{array}$ & $\begin{array}{c}\text { No-DR group } \\
\text { vs mild-NPDR } \\
\text { group }\end{array}$ \\
\hline \multicolumn{9}{|c|}{ RNFL thickness ( $\mu \mathrm{m})$} \\
\hline Average & $86.1 \pm 10.1$ & $86.4 \pm 10.2$ & $84.1 \pm 9.4$ & $91.2 \pm 7.3$ & 0.003 & 0.007 & 0.017 & 0.977 \\
\hline S & $104.3 \pm 19.4$ & $104.7 \pm 19.9$ & $101.1 \pm 15.5$ & $114.0 \pm 14.9$ & 0.005 & 0.009 & 0.030 & 1.000 \\
\hline $\mathrm{N}$ & $67.3 \pm 12.7$ & $67.7 \pm 13.3$ & $64.8 \pm 7.3$ & $66.9 \pm 8.6$ & 0.590 & 1.000 & 1.000 & 0.957 \\
\hline I & $113.2 \pm 16.2$ & $113.4 \pm 16.0$ & $112.4 \pm 17.7$ & $118.4 \pm 11.8$ & 0.111 & 0.139 & 0.423 & 1.000 \\
\hline $\mathrm{T}$ & $59.5 \pm 10.8$ & $59.7 \pm 10.6$ & $58.0 \pm 12.2$ & $65.4 \pm 9.9$ & 0.003 & 0.004 & 0.032 & 1.000 \\
\hline \multicolumn{9}{|c|}{ GC-IPL thickness $(\mu \mathrm{m})$} \\
\hline Average & $80.6 \pm 8.1$ & $80.7 \pm 8.1$ & $79.7 \pm 8.8$ & $85.3 \pm 9.9$ & 0.001 & 0.001 & 0.022 & 1.000 \\
\hline $\mathrm{SN}$ & $81.0 \pm 9.7$ & $81.0 \pm 9.8$ & $80.7 \pm 8.6$ & $86.4 \pm 5.8$ & 0.002 & 0.001 & 0.066 & 1.000 \\
\hline S & $81.7 \pm 8.9$ & $81.9 \pm 8.8$ & $79.9 \pm 10.0$ & $86.2 \pm 5.5$ & 0.003 & 0.007 & 0.015 & 0.942 \\
\hline ST & $80.3 \pm 7.6$ & $80.6 \pm 7.2$ & $78.5 \pm 9.8$ & $83.9 \pm 6.0$ & 0.004 & 0.014 & 0.016 & 0.734 \\
\hline IN & $79.3 \pm 9.6$ & $79.3 \pm 9.7$ & $79.5 \pm 8.7$ & $84.9 \pm 6.9$ & 0.001 & 0.001 & 0.079 & 1.000 \\
\hline I & $79.5 \pm 10.2$ & $79.4 \pm 10.4$ & $79.9 \pm 9.4$ & $84.3 \pm 6.3$ & 0.009 & 0.007 & 0.257 & 1.000 \\
\hline IT & $81.3 \pm 8.7$ & $81.6 \pm 8.4$ & $79.5 \pm 10.7$ & $85.8 \pm 6.1$ & 0.002 & 0.005 & 0.012 & 0.896 \\
\hline
\end{tabular}

Abbreviations: GC-IPL, ganglion cell-inner plexiform layer; I, inferior; IN, infero-nasal; IT, infero-temporal; mild-NPDR group, diabetes patients with signs of mild nonproliferative diabetic retinopathy (NPDR); N, nasal; no-DR group, diabetes patients without any sign of diabetic retinopathy (DR); RNFL, retinal nerve fiber layer; S, superior; SN, supero-nasal; ST, supero-temporal; T, temporal.

Values were compared by one-way analysis of variance (ANOVA), followed by Bonferroni post hoc test. Statistical significant $P$-values are written in bold. 
Table 3 Ganglion cell-inner plexiform layer and retinal nerve fiber layer thicknesses in diabetic patients and controls

\begin{tabular}{|c|c|c|c|c|c|c|c|}
\hline & \multicolumn{3}{|c|}{ Diabetic patients $(\mathrm{n}=150)$} & \multirow[t]{2}{*}{$\begin{array}{l}\text { Controls } \\
(\mathrm{n}=50)\end{array}$} & \multirow{2}{*}{$\begin{array}{c}\text { No OCT } \\
\text { defects group vs } \\
\text { Controls }\end{array}$} & \multirow{2}{*}{$\begin{array}{l}\text { OCT defects } \\
\text { group vs } \\
\text { Controls }\end{array}$} & \multirow{2}{*}{$\begin{array}{l}\text { No OCT defects } \\
\text { group vs OCT } \\
\text { defects group }\end{array}$} \\
\hline & $\begin{array}{l}\text { Overall } \\
\text { patients }\end{array}$ & $\begin{array}{l}\text { No OCT defects } \\
\text { group }(\mathrm{n}=88)\end{array}$ & $\begin{array}{c}\text { OCT defects group } \\
(\mathrm{n}=62)\end{array}$ & & & & \\
\hline \multicolumn{8}{|c|}{ RNFL thickness $(\mu \mathrm{m})$} \\
\hline Average & $86.1 \pm 10.1$ & $86.5 \pm 9.7$ & $85.6 \pm 10.6$ & $91.2 \pm 7.3$ & 0.015 & 0.006 & 1.000 \\
\hline $\mathrm{S}$ & $104.3 \pm 19.4$ & $105.0 \pm 15.0$ & $103.2 \pm 24.5$ & $114.0 \pm 14.9$ & 0.020 & 0.007 & 1.000 \\
\hline $\mathrm{N}$ & $67.3 \pm 12.7$ & $68.5 \pm 14.7$ & $65.6 \pm 9.1$ & $66.9 \pm 8.6$ & 1.000 & 1.000 & 0.423 \\
\hline I & $113.2 \pm 16.2$ & $113.6 \pm 15.8$ & $112.7 \pm 16.8$ & $118.4 \pm 11.8$ & 0.224 & 0.148 & 1.000 \\
\hline $\mathrm{T}$ & $59.5 \pm 10.8$ & $58.7 \pm 10.4$ & $60.6 \pm 11.3$ & $65.4 \pm 9.9$ & 0.001 & 0.055 & 0.864 \\
\hline \multicolumn{8}{|c|}{ GC-IPL thickness $(\mu \mathrm{m})$} \\
\hline Average & $80.6 \pm 8.1$ & $80.3 \pm 8.1$ & $81.0 \pm 8.2$ & $85.3 \pm 9.9$ & 0.001 & 0.010 & 1.000 \\
\hline $\mathrm{SN}$ & $81.0 \pm 9.7$ & $81.0 \pm 9.2$ & $81.0 \pm 10.4$ & $86.4 \pm 5.8$ & 0.003 & 0.006 & 1.000 \\
\hline$S$ & $81.7 \pm 8.9$ & $81.7 \pm 9.0$ & $81.6 \pm 8.9$ & $86.2 \pm 5.5$ & 0.008 & 0.012 & 1.000 \\
\hline ST & $80.3 \pm 7.6$ & $79.8 \pm 7.5$ & $81.0 \pm 7.7$ & $83.9 \pm 6.0$ & 0.004 & 0.092 & 0.939 \\
\hline IN & $79.3 \pm 9.6$ & $78.5 \pm 10.1$ & $80.4 \pm 8.7$ & $84.9 \pm 6.9$ & 0.001 & 0.026 & 0.572 \\
\hline I & $79.5 \pm 10.2$ & $79.4 \pm 10.2$ & $79.6 \pm 10.4$ & $84.3 \pm 6.3$ & 0.012 & 0.029 & 1.000 \\
\hline IT & $81.3 \pm 8.7$ & $80.7 \pm 8.9$ & $82.1 \pm 8.5$ & $85.8 \pm 6.1$ & 0.001 & 0.050 & 0.936 \\
\hline
\end{tabular}

Abbreviations: GC-IPL, ganglion cell-inner plexiform layer; I, inferior; IN, infero-nasal; IT, infero-temporal; mild-NPDR group, diabetes patients with signs of mild nonproliferative diabetic retinopathy (NPDR); N, nasal; no-DR group, diabetes patients without any sign of diabetic retinopathy (DR); RNFL, retinal nerve fiber layer; $\mathrm{S}$, superior; $\mathrm{SN}$, supero-nasal; $\mathrm{ST}$, supero-temporal; $\mathrm{T}$, temporal.

Values were compared by one-way analysis of variance (ANOVA), followed by Bonferroni post hoc test. Statistical significant $P$-values are written in bold.

a homogenous control group. Interestingly, these findings were also present in patients without any sign of DR compared with healthy controls, indicating this alteration occurs early in diabetes.

Neuroretinal degeneration is present in several neurological diseases, such as Alzheimer's and amyotrophic lateral sclerosis. ${ }^{11,14}$ Moreover, neuroretinal degeneration is distinctive and early in different optic nerve diseases, such as glaucoma. ${ }^{15}$ Interestingly, various studies showed that neuroretinal degeneration is also a retinal disease feature. Indeed, GC-IPL and RNFL thinning has been found in retinal illnesses, such as nonproliferative idiopathic macular telangiectasia type $2 \mathrm{~A}$, currently considered a neuroretinal disorder. ${ }^{16}$ These studies were possible because of SD-OCT. SD-OCT is widely used to image the retina, and recent advances in segmentation algorithms have led to the study of individual retinal layers with high resolution and good reproducibility. ${ }^{17-20}$

Several studies showed the early neuroretinal degeneration in diabetic patients. For example, several studies showed a decreased retinal ganglion cell (RGC) layer thickness in patients with type 1 diabetes. ${ }^{21}$ Moreover, in type 2 diabetic patients, neuroretinal alterations are supported both by a retinal function test, electroretinogram or microperimetry, ${ }^{3,4}$ and neuroretinal histological evaluation. ${ }^{22,23}$ To the best of our knowledge, only a few published studies exist testing GC-IPL and RNFL thicknesses in patients with no signs of DR using an imaging approach. ${ }^{24,25}$
In contrast to our results, Van Dijk HW et al ${ }^{24}$ showed no significant decrease in the GC-IPL and RNFL thickness values in patients affected by type 2 diabetes and without any signs of DR. However, this is probably secondary to the different SD-OCT types used (Cirrus and Topcon in our and Van Dijk HW's study, respectively) or to different patient group sizes (131 and 39 in our and Van Dijk HW's study, respectively).

We hypothesize that chronic hyperglycemia, even without clinically detectable microvascular complications, can negatively affect RGCs, leading to the functional impairment and death of RGCs and, consequently, a reduction of GC-IPL thickness and RNFL thickness. These are suggested by Barber et al, ${ }^{5}$ who showed increased apoptosis of retinal neural cells both in experimental diabetic rats and in diabetic patients. Increased apoptosis is probably due to the following: (i) neurofilament accumulation in RGC axons, related to changes in retrograde axonal transport; ${ }^{26}$ (ii) elevated levels of glutamate; (iii) increasing neurotoxic factors, ${ }^{27}$ and (iv) reactive changes in microglia. ${ }^{28}$

Moreover, comparing patients without abnormalities on the RNFL and GC-IPL analysis with healthy controls, we found a significant GC-IPL and RNFL thickness reduction. The latter feature supports the postulate that neuroretinal alterations occur early and are also present in patients with a normal SD-OCT analysis.

However, because all mild NPDR patients were affected by grade 20 Early Treatment Diabetic Retinopathy Study 
Table 4 Correlation among diabetes duration, $\mathrm{HbA} 1 \mathrm{c}$ value, RNFL thickness, and GC-IPL thickness in diabetic patients

\begin{tabular}{|c|c|c|}
\hline & Diabetes duration & HbA1c value \\
\hline \multicolumn{3}{|l|}{ RNFL thickness ( $\mu \mathrm{m})$} \\
\hline \multicolumn{3}{|l|}{ Average } \\
\hline Pearson Correlation & -0.152 & 0.162 \\
\hline$P$-value & 0.072 & 0.048 \\
\hline \multicolumn{3}{|l|}{$\mathrm{S}$} \\
\hline Pearson Correlation & -0.134 & 0.152 \\
\hline$P$-value & 0.114 & 0.065 \\
\hline \multicolumn{3}{|l|}{$\mathrm{N}$} \\
\hline Pearson Correlation & -0.017 & -0.024 \\
\hline$P$-value & 0.841 & 0.774 \\
\hline \multicolumn{3}{|l|}{ I } \\
\hline Pearson Correlation & -0.086 & 0.162 \\
\hline$P$-value & 0.309 & 0.048 \\
\hline \multicolumn{3}{|l|}{$\mathrm{T}$} \\
\hline Pearson Correlation & -0.164 & 0.101 \\
\hline$P$-value & 0.052 & 0.222 \\
\hline \multicolumn{3}{|l|}{ GC-IPL thickness $(\mu \mathrm{m})$} \\
\hline \multicolumn{3}{|l|}{ Average } \\
\hline Pearson Correlation & -0.137 & 0.081 \\
\hline$P$-value & 0.106 & 0.329 \\
\hline \multicolumn{3}{|l|}{ SN } \\
\hline Pearson Correlation & -0.146 & 0.055 \\
\hline$P$-value & 0.083 & 0.509 \\
\hline \multicolumn{3}{|l|}{ S } \\
\hline Pearson Correlation & -0.145 & 0.074 \\
\hline$P$-value & 0.086 & 0.371 \\
\hline \multicolumn{3}{|l|}{ ST } \\
\hline Pearson Correlation & -0.178 & 0.067 \\
\hline$P$-value & 0.035 & 0.421 \\
\hline \multicolumn{3}{|l|}{ IN } \\
\hline Pearson Correlation & -0.057 & 0.099 \\
\hline$P$-value & 0.503 & 0.228 \\
\hline \multicolumn{3}{|l|}{ I } \\
\hline Pearson Correlation & -0.088 & 0.071 \\
\hline$P$-value & 0.299 & 0.391 \\
\hline \multicolumn{3}{|l|}{ IT } \\
\hline Pearson Correlation & -0.121 & 0.051 \\
\hline$P$-value & 0.152 & 0.536 \\
\hline
\end{tabular}

Abbreviations: GC-IPL, ganglion cell-inner plexiform layer; I, inferior; IN, infero-nasal; IT, infero-temporal; N, nasal; RNFL, retinal nerve fiber layer; $\mathrm{S}$, superior; $\mathrm{SN}$, supero-nasal; ST, supero-temporal; $\mathrm{T}$, temporal. Statistical significant $P$-values are written in bold.

(only microaneurisms as pathological signs), we did not adjust the results for pathological signs.

The absence of correlation between the thinning of GC-IPL and RNFL and diabetes duration was previously reported by Van Dijk et al ${ }^{24}$ This feature is likely because the disease process is unclear in patients with type 2 diabetes, because glucose metabolism can be altered years before diabetes diagnosis. Therefore, the possible correlation between the thinning of GC-IPL and RNFL and the duration of disease is not precise.

The presence of a direct correlation between the GC-IPL and RNFL average thickness values and HbA1c level is in the opposite of many studies. Nevertheless, we predict that this result is secondary to the higher Hba1c values in patients with a $<1$-year diabetes diagnosis compared with all other patients. Indeed, the correlation was no longer present for patients with a $>1$-year diabetes diagnosis.

Our study has several limitations. The main limitation is the method used. Automated segmentation, although reproducible, has shown test-retest variability in testing GC-IPL and RNFL thickness. ${ }^{17,19,20}$ However, SD-OCT imaging remains the most reproducible tool in testing these two layers. Another important limitation is that the sample size is relatively small. However, the strict inclusion criteria for patients should be considered. Finally, another limitation is that we did not measure the axial length to avoid an invasive exam for the patient. However, both the axial length and the refractive error influence the GC-IPL and RNFL measurements. ${ }^{29}$ Nevertheless, one should consider the following: (1) the low variability of refractive error in the enrolled subjects; (2) the refractive error mean \pm SD is very similar in the different groups; and (3) no patient underwent refractive surgery.

In conclusion, we confirmed the role of SD-OCT for the evaluation of asymptomatic diabetes patient without any sign of DR and also that neuroretinal degeneration is early, preceding microvascular damages. Further studies are necessary to understand whether ganglion cell neuroretinal degeneration and microvascular damages are pathogenically linked and whether neuroretinal degeneration represents a target in diabetes treatment to prevent DR.

\section{Summary}

What was known before

- Retinal functional impairment may occur early in the course of diabetes, also in patients without any sign of DR, suggesting a role of neuroretinal damage in the DR pathogenesis.

- Autopsy study showed neuroretinal damage is earlydiabetic patient retina.

\section{What this study adds}

- We demonstrated a significantly reduced ganglion celliere plexiform layer and retinal serve fiber player thickness values in patients without any sign of DR.

- Our data confirmed neuroretinal alterations are early in diabetes, preceding microvascular damages.

\section{Conflict of interest}

The authors declare no conflict of interest.

\section{References}

1 Fong DS, Aiello LP, Ferris FL, Klein R. Diabetic retinopathy. Diabetes Care 2004; 27: 2540-2553. 
2 Antcliff RJ, Marshall J. The pathogenesis of edema in diabetic maculopathy. Semin Ophthalmol 1999; 14: 223-232.

3 De Benedetto U, Querques G, Lattanzio R, Borrelli E, Triolo G, Maestranzi $G$ et al. Macular dysfunction is common in both type 1 and type 2 diabetic patients without macular edema. Retina 2014; 34: 2171-2177.

4 Bearse MA, Ozawa GY. Multifocal electroretinography in diabetic retinopathy and diabetic macular edema. Curr Diab Rep 2014; 14: 526.

5 Barber AJ, Lieth E, Khin SA, Antonetti DA, Buchanan AG, Gardner TW et al. Neural apoptosis in the retina during experimental and human diabetes. Early onset and effect of insulin. J Clin Invest 1998; 102: 783-791.

6 Biallosterski C, van Velthoven MEJ, Michels RPJ, Schlingemann RO, DeVries JH, Verbraak FD. Decreased optical coherence tomography-measured pericentral retinal thickness in patients with diabetes mellitus type 1 with minimal diabetic retinopathy. Br J Ophthalmol 2007; 91: $1135-1138$.

7 Bronson-Castain KW, Bearse MA, Neuville J, Jonasdottir S, King-Hooper B, Barez S et al. Adolescents with Type 2 diabetes: early indications of focal retinal neuropathy, retinal thinning, and venular dilation. Retina 2009; 29: 618-626.

8 Nilsson M, von Wendt G, Wanger P, Martin L. Early detection of macular changes in patients with diabetes using Rarebit Fovea Test and optical coherence tomography. Br J Ophthalmol 2007; 91: 1596-1598.

9 Cabrera DeBuc D, Somfai GM. Early detection of retinal thickness changes in diabetes using optical coherence tomography. Med Sci Monit 2010; 16: MT15-MT21.

10 Wilkinson CP, Ferris FL, Klein RE, Lee PP, Agardh CD, Davis $\mathrm{M}$ et al. Proposed international clinical diabetic retinopathy and diabetic macular edema disease severity scales. Ophthalmology 2003; 110: 1677-1682.

11 Bambo MP, Garcia-Martin E, Otin S, Pinilla J, Larrosa JM, Polo $\mathrm{V}$ et al. Visual function and retinal nerve fibre layer degeneration in patients with Alzheimer disease: correlations with severity of dementia. Acta Ophthalmol 2015; 93(6): e507-e508.

12 Yu J-G, Feng Y-F, Xiang Y, Huang JH, Savini G, Parisi V et al. Retinal nerve fiber layer thickness changes in Parkinson disease: a meta-analysis. PLoS One 2014; 9: e85718.

13 Mwanza J-C, Oakley JD, Budenz DL, Chang RT, Knight OJ, Feuer WJ et al. Macular ganglion cell-inner plexiform layer: automated detection and thickness reproducibility with spectral domain-optical coherence tomography in glaucoma. Invest Ophthalmol Vis Sci 2011; 52: 8323-8329.

14 Ringelstein M, Albrecht P, Südmeyer M, Harmel J, Müller AK, Keser $\mathrm{N}$ et al. Subtle retinal pathology in amyotrophic lateral sclerosis. Ann Clin Transl Neurol 2014; 1: 290-297.

15 Rao HL, Yadav RK, Addepalli UK, Begum VU, Senthil S, Choudhari NS et al. Comparing spectral-domain optical coherence tomography and standard automated perimetry to diagnose glaucomatous optic neuropathy. J Glaucoma 2015; 24(5): e69-e74.

16 Chhablani J, Rao HL, Begum VU, Jonnadulla GB, Goud A, Barteselli G. Retinal ganglion cells thinning in eyes with non- proliferative idiopathic macular telangiectasia type 2A. Invest Ophthalmol Vis Sci 2015; 56(2): 1416-1422.

17 Carpineto P, Nubile M, Agnifili L, Toto L, Aharrh-Gnama A, Mastropasqua $\mathrm{R}$ et al. Reproducibility and repeatability of CirrusTM HD-OCT peripapillary retinal nerve fibre layer thickness measurements in young normal subjects. Ophthalmologica 2012; 227: 139-145.

18 Pierro L, Gagliardi M, Iuliano L, Ambrosi A, Bandello F. Retinal nerve fiber layer thickness reproducibility using seven different OCT instruments. Invest Ophthalmol Vis Sci 2012; 53: 5912-5920.

19 Garvin MK, Lee K, Burns TL, Abràmoff MD, Sonka M, Kwon YH. Reproducibility of SD-OCT-based ganglion cell-layer thickness in glaucoma using two different segmentation algorithms. Invest Ophthalmol Vis Sci 2013; 54: 6998-7004.

20 Leung CK-S, Cheung CY-L, Weinreb RN, Qiu Q, Liu S, $\mathrm{Li} \mathrm{H}$ et al. Retinal nerve fiber layer imaging with spectraldomain optical coherence tomography: a variability and diagnostic performance study. Ophthalmology 2009; 116: 1257-1263. 1263.e1-2.

21 Van Dijk HW, Verbraak FD, Kok PHB, Garvin MK, Sonka M, Lee $\mathrm{K}$ et al. Decreased retinal ganglion cell layer thickness in patients with type 1 diabetes. Invest Ophthalmol Vis Sci 2010; 51: 3660-3665.

22 Barber AJ. A new view of diabetic retinopathy: a neurodegenerative disease of the eye. Prog Neuropsychopharmacol Biol Psychiatry 2003; 27: 283-290.

23 Lieth E, Gardner TW, Barber AJ, Antonetti DA. Penn State Retina Research Group. Retinal neurodegeneration: early pathology in diabetes. Clin Experiment Ophthalmol 2000; 28: 3-8.

24 Van Dijk HW, Verbraak FD, Kok PHB, Stehouwer M, Garvin MK, Sonka M et al. Early neurodegeneration in the retina of type 2 diabetic patients. Invest Ophthalmol Vis Sci 2012; 53: 2715-2719.

25 Rodrigues EB, Urias MG, Penha FM, Badarò E, Novais E, Mereilles $\mathrm{R}$ et al. Diabetes induces changes in neuroretina before retinal vessels: a spectral-domain optical coherence tomography study. Int J Retin Vitr 2015; 1: 4.

26 Gastinger MJ, Kunselman AR, Conboy EE, Bronson SK, Barber AJ. Dendrite remodeling and other abnormalities in the retinal ganglion cells of Ins2 Akita diabetic mice. Invest Ophthalmol Vis Sci 2008; 49: 2635-2642.

27 Villarroel M, Ciudin A, Hernández C, Simó R. Neurodegeneration: an early event of diabetic retinopathy. World $\mathrm{J}$ Diabetes 2010; 1: 57-64.

28 Antonetti DA, Klein R, Gardner TW. Diabetic retinopathy. N Engl J Med 2012; 366: 1227-1239.

29 Ueda K, Kanamori A, Akashi A, Tomioka M, Kawaka Y, Nakamura M. Effects of Axial Length and Age on Circumpapillary Retinal Nerve Fiber Layer and Inner Macular Parameters Measured by 3 Types of SD-OCT Instruments. J Glaucoma; e-pub ahead of print 14 January 2015; doi:10.1097/IJG.0000000000000216. 